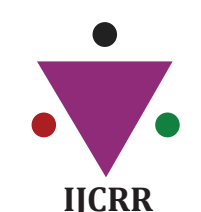

IJCRR

Section: Healthcare

ISI Impact Factor

(2019-20): 1.628

IC Value (2019): 90.81

SJIF (2020) $=7.893$

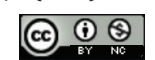

Copyright@IJCRR

\section{A Comparative Clinical Evaluation of Access Cavity Preparation Using Dental Operating Microscope and Conventional Preparation for Conservation of Tooth Structure}

\author{
Manoj Chandak ${ }^{1}$, Pradnya Nikhade ${ }^{1}$, P. Sindhu², Anuja Ikhar ${ }^{3}$, Rakhi Chandak ${ }^{4}$, \\ Abhilasha Dass ${ }^{2}$, Nidhi Motwani5
}

\begin{abstract}
'Professor, Department of Conservative Dentistry and Endodontics, Sharad Pawar Dental College \& Hospital, Datta Meghe lnstitute of Medical Sciences (Deemed to be University) Sawangi (Meghe) Wardha, Maharashtra, India; '2Lecturer, Department of Conservative Dentistry and Endodontics, Sharad Pawar Dental College \& Hospital, Datta Meghe Institute of Medical Sciences (Deemed to be University) Sawangi (Meghe) Wardha, Maharashtra, India; ${ }^{A}$ Associate Professor, Department of Conservative Dentistry and Endodontics, Sharad Pawar Dental College \& Hospital, Datta Meghe Institute of Medical Sciences (Deemed to be University) Sawangi (Meghe) Wardha, Maharashtra, India; ${ }^{4}$ Department of Oral Medicine and Radiology, Swargiya Dadasaheb Kalmegh Smruti Dental College and Hospital, Nagpur, Maharashtra, India; ${ }^{5}$ Postgraduate Student, Department of Conservative Dentistry and Endodontics, Sharad Pawar Dental College \& Hospital, Datta Meghe Institute of Medical Sciences (Deemed to be University) Sawangi (Meghe) Wardha, Maharashtra, India.
\end{abstract}

\title{
ABSTRACT
}

Background: The outcome of endodontic therapy is associated with eradication of micro-organisms from the root canal system and avoidance of re-infection. Optical magnifications have extended the horizons of dental treatment. Recently, illumination and magnification have established to help provide precise anatomic details. The canal orifices can effortlessly be identified under a microscope than with surgical loupes or the naked eye.

Objective: The comparison of the conservation of tooth structure during access cavity preparation under the microscope with the conventional method.

Methods: Thirty patients were selected for whom root canal treatment where indicated. The patients were divided into two groups Group 1 - Access cavity prepared conventionally and Group 2 - Access cavity preparation under the microscope. The measurement between intercanal orifice was done using Vernier callipers.

Results: In the present study, there was a reduction in measurement between mesiobuccal to the distal orifice and mesiobuccal to mesiolingual orifice but it was not statistically significant. There was a reduction in measurement from mesiolingual to distal orifice. The reduction was statistically significant $(p=0.018)$ Less amount of tooth structure is removed therefore allowing for conservation of tooth structure resulting in improved strength tooth strength, which is clinically important.

Conclusion: The conservative endodontic cavity (CEC) preparation to reduce the loss of tooth and preservation some of the roofs of the pulp chamber and peri-cervical dentin was reported in the literature.

Key Words: Conservative endodontic cavity, Pre-operative radiograph, Distal canal orifice

\section{INTRODUCTION}

The mandibular first molar poses many anatomic challenges and most frequently endodontically treated tooth, but it also. The access cavity has an external outline form evolving from internal pulpal anatomy of the tooth. First mandibular molar generally has 2 roots and 3 root canals. However, due to genetic, ethnic and gender varieties, a wider range of anatomi- cal, as well as morphological deviations, can be encountered. The difficulty lies in the location of the number and position of orifices on pulp chamber floors. ${ }^{1}$ This is particularly correct when the tooth is excessively restored, calcified or malpositioned. The commonly used method to evaluate the morphology of root canal involves staining of the root canal and tooth clearing, plastic injection, conventional and digital radiography and radiopaque gel infusion and radiography. ${ }^{2}$

Corresponding Author:

Nidhi Motwani, Postgraduate Student, Department of Conservative Dentistry and Endodontics, Sharad Pawar Dental College \& Hospital, Datta Meghe Institute of Medical Sciences (Deemed to be University) Sawangi (Meghe) Wardha, Maharashtra, India; Email: nidhimotwani20@gmail.com ISSN: 2231-2196 (Print) ISSN: 0975-5241 (Online)

Received: $31.07 .2020 \quad$ Revised: 03.10 .2020 Accepted: 14.11 .2020 Published: 16.01 .2021 
The clinician should be clear and thorough about root canal morphology of tooth being treated since it can affect the treatment outcome ${ }^{3}$ The outcome of endodontic therapy is associated with eradication of micro-organisms from root canal system and avoidance of re-infection. ${ }^{4}$ Optical magnifications have extended the horizons of dental treatment. Recently, illumination and magnification have established to help provide precise anatomic details. The canal orifices can effortlessly be identified under a microscope than with surgical loupes or the naked eye..$^{5}$ Based on lens system microscope can be classified into the simple or compound microscope. The lens system varies from double to 2 planoconvex. Based on magnification, the microscope can work under low, mid-range and high magnification. The microscope has higher magnification in the range of X2.5 to X30 with better illumination using fibre optic technology. The reflecting microscope wherein an opaque substance requires light that reflects from the object to the lenses in an optic tube. ${ }^{6}$ One of the uses of magnification in endodontics is the conservation of access opening. ${ }^{7}$ So the purpose of the present study was the comparison of the conservation of tooth structure during access cavity preparation under the microscope with the conventional method. The research question was does magnification using Dental operating microscope aid in the conservation of tooth during access cavity preparation in endodontic treatment as compared to the conventional method. ${ }^{8,9}$

In the present study, we aimed to assess the conservation of tooth structure using the conventional method in root canal treatment. Further, we assessed the conservation of tooth structure using a dental operating microscope in root canal treatment and compared the conservation of tooth structure using the conventional technique with dental operating microscope in root canal treatment.

\section{MATERIALS \& METHODS}

The study was performed at Department of Conservative Dentistry and Endodontics Sharad Pawar Dental College \& Hospital, Sawangi, Wardha after getting approved by ethical committee (IEC reference no. DMIMS(DU)/IEC/Dec2019/8616).

\section{Selection of data}

Thirty patients were selected for whom root canal treatment where indicated. Patients were explained about the procedure in detail and informed consents were obtained. Mandibular $1^{\text {st }}$ molars were selected for the study.

\section{Inclusion Criteria}

- Teeth with caries involving enamel, dentin and pulp requiring root canal treatment.
- Teeth with completely developed roots.

\section{Exclusion criteria}

- Teeth with evident periapical pathology, tooth mobility, root resorption.

- Patients with medical conditions,

- Pregnant, lactating women.

Informed written consents were obtained from each patient before treatment.

\section{Procedure}

\section{Radographs}

A pre-operative radiograph (IOPA) was taken from different angulation to study the dimensions of the pulp chamber, number of canals and length of the root. This helps in the correct approach of access cavity preparation. ${ }^{10}$

\section{Access opening}

Patch test was performed to check for allergy for a local anaesthetic solution. After proper palpation of anatomical landmarks, Local anaesthetic was administered into the retromolar area to block the Inferior Alveolar nerve. Mandibular first molar was isolated with a rubber dam. The patients were divided into two groups ( Table 1)

\begin{tabular}{|c|c|c|}
\hline Sr No. & GROUPS & METHOD \\
\hline 1 & Group $1(n=15)$ & $\begin{array}{l}\text { Access Cavity prepared conven- } \\
\text { tionally }\end{array}$ \\
\hline 2 & Group $2(n=15)$ & $\begin{array}{l}\text { Access cavity prepared under a } \\
\text { microscope }\end{array}$ \\
\hline
\end{tabular}

\section{For Group 1}

Conventional cavity preparation was done using \#4 carbide or round diamond no 4 or \#557 tapered fissure bur was used. The bur was advanced through the central fossa until a drop was felt. The drop indicates that the pulp chamber is explored. Safely ended bur was used to restrict the depth to avoid cutting of floor of the tooth. All canals orifices were located with DG16 explorer and enlarged with G.G drill. Measurement - Measurement between intercanal orifice was done using Vernier callipers and listed in the table.

\section{For Group 2}

Standardized access cavity preparation was done under an operating microscope with $12 \mathrm{X}$ magnification. The measurement between intercanal orifice was done using Vernier callipers and listed in the table

\section{Statistical Analysis}

The data was collected and analyzed using by comparing the mean and by Wilcoxon sign-rank test. 


\section{RESULTS}

The strength of the tooth is directly proportional to the amount of removal of dentin. More the removal of dentin more will be the tooth structure loss and this causes weakening of the tooth. The destruction was evaluated by measuring the distance between the orifices using Vernier callipers.

Table 1: The mean values of the measurements of the
inter orifice distance
\begin{tabular}{llccc} 
Sr. No. Groups & Mean & Std. Deviation & Sig. \\
1. & MicroMBD & 3.03 & .44 & 0.646 (NS) \\
& ConMBD & 3.06 & .42 & \\
2. & MicroMLD & 3.30 & .45 & $0.018^{*}$ \\
3. ConMLD & 3.40 & .42 & \\
& MicroMBML & 1.80 & .25 & $0.294(\mathrm{NS})$ \\
\hline
\end{tabular}

Graph represent mean of distance between mesiobuccal to distal canal orifice(mbd): mesiolingual to distal canal orifice (mld) and mesiobuccal and mesiolungual canal orifice ( $\mathrm{mbml})$ in access cavity prepared under microscope and conventional preparation.

\section{DISCUSSION}

The scientific-technical advance in endodontics in the last years has been increasing the incidence of success of the treatment of root canals. The absence of spontaneous and provoked painful symptomatology, hermetic sealing, and dental element rehabilitated in masticatory function, and the healing of the apical and periapical tissue are clinical criteria of success in endodontic therapy. ${ }^{11}$ An endodontically treated teeth, one of the failures is the fracture of the tooth. The conservative access cavity preparation was anticipated to diminish fracture possibility of endodontically treated teeth. ${ }^{12}$ Since the 2000 s in clinical practice, dental operating microscopes have been utilized as the best reliable aid. The microscopes aid in recognizing then dentinal coverage present over the orifice. Further, this dentinal coverage could be removed accurately with an ultrasonic instrument which would help in recognization of canal orifice. ${ }^{5}$ Further the microscope also helps to analyze the dentinal wall for the carious lesion. ${ }^{13}$ As stated by Dr. Carr "You cannot treat what you cannot see". The conservative endodontic cavity (CEC) preparation to reduce the loss of tooth and preservation some of the roofs of the pulp chamber and peri-cervical dentin was reported in the literature. ${ }^{14}$ Therefore, the removal of dentin was evaluated by measuring the distance between the orifices using vernier callipers.

In the present study, there was a reduction in measurement between mesiobuccal to the distal orifice and mesiobuccal to mesiolingual orifice but it was not statistically significant. There was a reduction in measurement from mesiolingual to distal orifice. The reduction was statistically significant $(p=0.018)$ Less amount of tooth structure is removed therefore allowing for conservation of tooth structure resulting in improved strength tooth strength, which is clinically important. ${ }^{15}$ Paul Krasner and Henry Rankow in 2004 stated that microscope enhances the capability of the location of the canal orifices/ number on pulpal floor. If there is the presence of anatomical landmarks, then the task of locating orifices will be more systematic and, consequently, with superior certainty. The law of change in colour aids in location canal orifice. However, the difference in colour between the floor and walls of the pulp chamber is stressful to differentiate with the unaided eye. Also, the walls of the pulp cavity were constantly concentric to the external surface of the tooth at the level of the CEJ, Also at the level of the CEJ, the distance from the external surface of the tooth to the wall of the pulpal chamber was equal all through the circumference. ${ }^{16}$ Magnification with illumination provided with the microscope is helpful to prevent the stress on the human eye. The microscope helps in the determination of the dentinal map that is of key importance in locating the canal. Carr et al reported that using the DOM clinically, their ability to identify MB2 canals in maxillary $1^{\text {st }}$ molars increased by $20 \% .{ }^{17} \mathrm{Ac}-$ cording to Carr et al, the frequency of detection of the MB2 canal was $17 \%$ without magnification and was increased to $63 \%$ using loupes and $71 \%$ using the DOM. According to Enrique Merino, the microscope increases the ability of the dentist to locate and negotiate the hidden canal. ${ }^{18}$ The use of the microscope increases the predictability of the treatment. Ultimately this increases the success rate of the endodontic treatment. Mittal et al concluded that the number of orifices detected increased with increasing magnification. ${ }^{18}$

\section{CONCLUSION}

The art of dentistry is based on precision. The unaided eye can only see up to canal orifice, Microscope function as $3^{\text {rd }}$ eye for Endodontist. This study showed that access cavity prepared under dental operating microscope conserved more tooth structure as compared to the conventional technique. A statistically significant difference was noted with mesio lingual orifice to distal orifice in conventional access cavity preparation as compared to the dental operating microscope when the inter-orifice distance was measured. We can recommend that the access cavity preparation should be performed under magnification with the help of a dental operating microscope to retain the strength of dentin and prevent tooth fracture that will help in the long period success of the procedure. 


\section{ACKNOWLEDGEMENT}

Authors acknowledge the immense help received from the scholars whose articles are cited and included in references to this manuscript. The authors are also grateful to authors/ editors/publishers of all those articles, journals and books from where the literature for this article has been reviewed and discussed.

\section{Conflict of Interest: Nil}

\section{Source of Funding: Nil}

\section{REFERENCES}

1. Karunakaran JV, Samuel LS, Rishal Y, Joseph MD, Suresh KR, Varghese ST. Root canal configuration of human permanent mandibular first molars of an Indo-Dravidian populationbased in Southern India: An in vitro study. J Pharm Alli Sci 2017 Nov;9(1): S68.

2. Khlaghi NM, Khalilak Z, Vatanpour M, Mohammadi S, Pirmoradi S, Fazlyab M, Safavi K. Root canal anatomy and morphology of mandibular first molars in a selected Iranian population: an in vitro study. Iranian Endod J 2017;12(1):87.

3. Nixdorf DR, Moana-Filho EJ, Law AS, McGuire LA, Hodges JS, John MT. Frequency of persistent tooth pain after root canal therapy: a systematic review and meta-analysis. J Endod 2010 Feb; 36(2):224-230.

4. Fabra-Campos H. Three canals in the mesial root of mandibular first permanent molars: a clinical study. Int Endod J 1989 Jan; 22(1):39-43.

5. Gogiya RJ, Chandak MG, Modi RR, Bhutda P, Kela S, Chandak RM. Magnification in dentistry: A review. Int J Appl Dent Sci 2018;4(2):89-93.

6. Pecora G, Andreana S. Use of the dental operating microscope in endodontic surgery. Oral Surg Oral Med Oral Pathol Oral Radiol Endod 1993;75:751-758.
7. Low JF, Dom TN, Baharin SA. Magnification in endodontics: A review of its application and acceptance among dental practitioners. Eur J Dentistry 2018 Oct;12(04):610-616.

8. Reeh ES, Messer HH, Douglas WH. Reduction in tooth stiffness as a result of endodontic and restorative procedures. J Endod 1989;15(11):512-516.

9. Ayse DK, Bulem UK, Erhan S. Determination of canal orifice co-ordinates and MB2 incidence of maxillary first molars in a Turkish subpopulation. Odontologica Scandinavica. 2014; 72: 354-361.

10. Corsentino G, Pedullà E, Castelli L, Liguori M, Spicciarelli V, Martignoni M, Grandini S. Influence of Access Cavity Preparation and Remaining Tooth Substance on Fracture Strength of Endodontically Treated Teeth. J Endodontics 2018;3(6):543-547.

11. Osman IA, Ahmed H. The Effect of Access Cavity Design on Fracture Resistance of Endodontically Treated First Molars: In Vitro Study. Dove Press 2017; 5(9):443-451.

12. Özyürek T, Ülker Ö, Demiryürek EÖ, Yilmaz F. The Effects of Endodontic Access Cavity Preparation Design on the Fracture Strength of Endodontically Treated Teeth: Traditional Versus Conservative Preparation. J Endod 2018;44(5):800-805.

13. Rathi NV, Chandak MG, Mude GA. Comparative Evaluation of Dentinal Caries in Restored Cavity Prepared By Galvanic and Sintered Burs. Contemp Clin Denst 2018;9(1):S23-27.

14. Saygili G, Uysal B, Omar B. Evaluation of the relationship between endodontic access cavity types and secondary mesiobuccal canal detection. BMC Oral Health 2018;18:121.

15. Mukherjee P, Patel A, Chandak M, Kashikar R. Minimally Invasive Endodontics a Promising Future Concept: A Review Article. Int J Sci Study 2017;5(1):245-251.

16. Krasner P. Rankow HJ. Anatomy of Pulp Chamber Floor. J Endod 2004;30(1): 5-16.

17. Carr G.B, Murgel C. The use of the operating microscope in Endodontics. J Dental Clin 2010; 54(2;191-214.

18. Endodontic Microsurgery, Ouintessence Company Ltd 2009. 